\title{
Effect of Neutral Grounding Protection Methods for Compensated Wind/PV Grid-Connected Hybrid Power Systems
}

\author{
Nurettin Çetinkaya ${ }^{1}$ and Farhana Umer $^{2}$ \\ ${ }^{1}$ Nurettin ÇETINKAYA, Electrical-Electronics Engineering Department, Selçuk University, Konya, Turkey \\ ${ }^{2}$ Farhana UMER, Electrical Engineering Department, The Islamia University of Bahawalpur Pakistan, Bahawalpur, Pakistan \\ Correspondence should be addressed to Nurettin Çetinkaya; ncetinkaya@selcuk.edu.tr
}

Received 3 June 2017; Revised 5 September 2017; Accepted 9 October 2017; Published 19 November 2017

Academic Editor: Leonardo Sandrolini

Copyright (C) 2017 Nurettin Çetinkaya and Farhana Umer. This is an open access article distributed under the Creative Commons Attribution License, which permits unrestricted use, distribution, and reproduction in any medium, provided the original work is properly cited.

\begin{abstract}
The effects of the wind/PV grid-connected system (GCS) can be categorized as technical, environmental, and economic impacts. It has a vital impact for improving the voltage in the power systems; however, it has some negative effects such as interfacing and fault clearing. This paper discusses different grounding methods for fault protection of High-voltage (HV) power systems. Influences of these grounding methods for various fault characteristics on wind/PV GCSs are discussed. Simulation models are implemented in the Alternative Transient Program (ATP) version of the Electromagnetic Transient Program (EMTP). The models allow for different fault factors and grounding methods. Results are obtained to evaluate the impact of each grounding method on the 3-phase short-circuit fault (SCF), double-line-to-ground (DLG) fault, and single-line-to-ground (SLG) fault features. Solid, resistance, and Petersen coil grounding are compared for different faults on wind/PV GCSs. Transient overcurrent and overvoltage waveforms are used to describe the fault case. This paper is intended as a guide to engineers in selecting adequate grounding and ground fault protection schemes for HV, for evaluating existing wind/PV GCSs to minimize the damage of the system components from faults. This research presents the contribution of wind/PV generators and their comparison with the conventional system alone.
\end{abstract}

\section{Introduction}

Sustainable energy sources (SESs) are about using energy wisely and using energy generated from clean sources and clean technologies. The larger number of SESs needs new schemes to improve or maintain the power quality and stability [1]. A grid interface with wind/PV farms improves system reliability [2].

SESs have direct influence on the integration of wind/PV due to changeable and uncertain condition in wind speed, solar irradiance, and location. The SCF value is a basic feature for a safe and protective system. SCF magnitude is most significant for coupling location to the GCS which must not increase the designed value $[3,4]$. The instability of SCF current sharing by distributed generation (DG) is a significant constraint in integrating the DG to the conventional system $[5,6]$. Transients occur in the power system due to various reasons such as faults, switch closing and opening, or lightning strikes $[7,8]$. The sudden connection of induction generators (IGs) to the busbars results in altering the transient current that also impacts the power quality [9].

Grounded systems have many advantages as compared to ungrounded systems [10-12]. The grounding methods have small impact when a system runs at normal operation but become effective and significant when fault occurs to an overhead line (OHL). The suitable grounding solution is achieved through calculations and simulations of the system under fault condition [13]. Resonant grounding support extinguished the fault arc in OHL for about 80 percent of temporary ground faults [14].

Many researchers previously discussed different types of neutral grounding, SLG fault, and fault location and detection in middle-voltage (MV) or HV conventional systems such as Al-Zyoud et al. who worked on Jordanian MV distribution systems (DSs) by using various techniques for grounding. Impacts of these earthing methods 


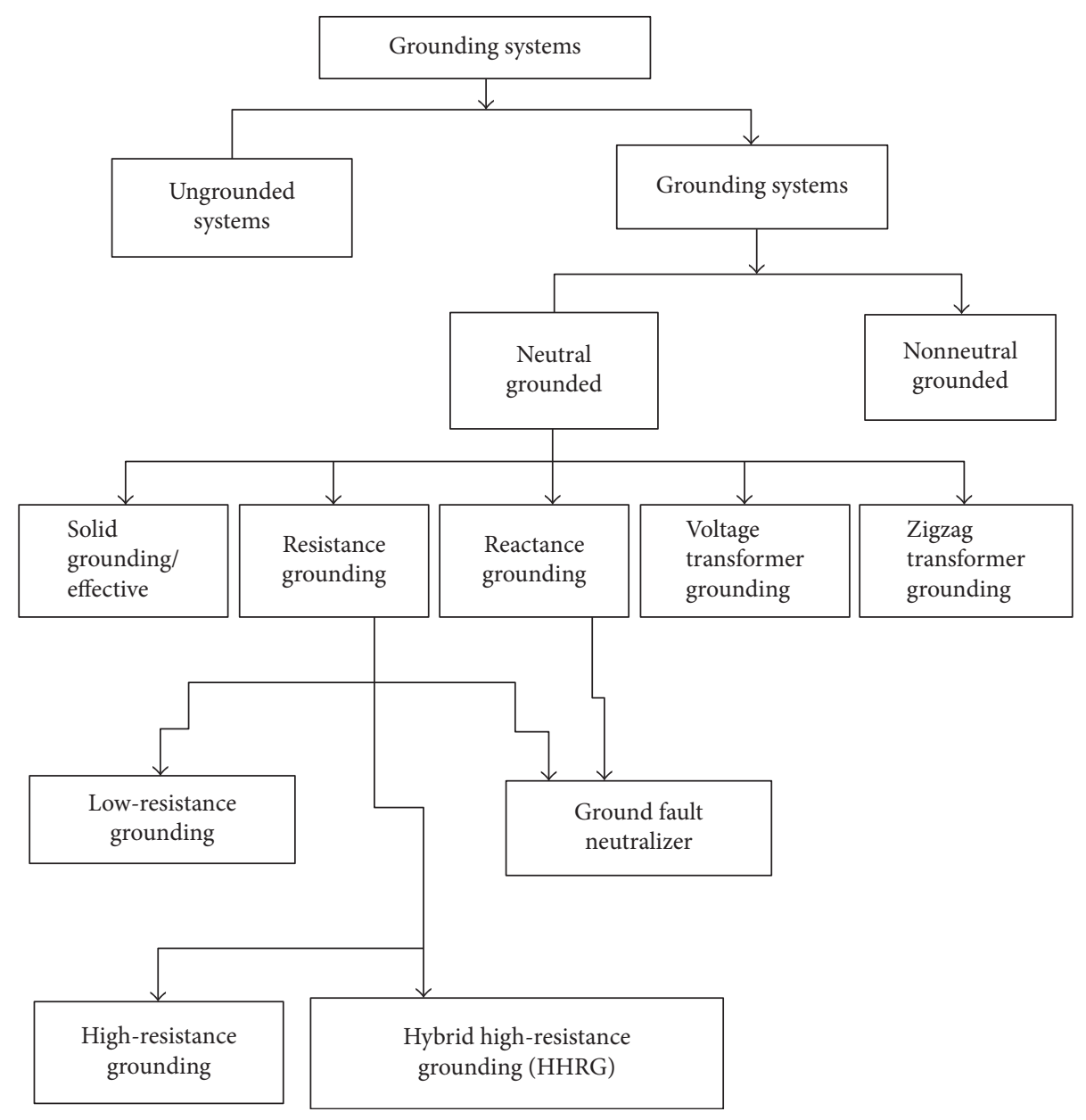

Figure 1: Types of grounding systems.

on the SLG fault are discussed [13]. Jacob and Nithiyanthan in their paper emphasized on the type of grounding based on rated voltage of the faulted network [15]. Pillai et al. in their paper discuss ground fault protection techniques for the MV stator of a generator and their merits and demerits [16]. Bapat et al. worked on application when the DSs considered multiple sources operating in parallel. Hybrid grounding was proposed for low and medium voltage systems [17]. In [18-21], the authors analyzed various types of neutral grounding. In $[22,23]$, the authors examined the voltage and current behaviour under fault condition for various parameters of systems. An isolated neutral system has low-value fault current. Many researchers created protection algorithms for compensation [24-28]. Overvoltage generated by single-line-to-ground (SLG) faults by using Petersen coil for MV systems is given in [29]. In [30], the authors analyzed the effect of neutral grounding techniques on the features of fault in $\mathrm{Al} \mathrm{Ain}$ DS in UAE. In [31], a few experimental fault cases were examined under various conditions on a DS in Czech Republic with Petersen coil grounding. In [32], the authors analyzed and compared the features of earth fault in MV systems, that is, $20 \mathrm{kV}$ with high-impedance grounding. The results are based on the evaluation of real case recordings, achieved in 3 years.
Most of the researchers worked on MV networks and during the SLG fault on the conventional system, but no one worked on HV networks and during short-circuit faults (SCFs). In this paper, the authors worked on wind/PV GCSs under the SCF and will study the contribution of wind/PV generators under SCF condition and their comparison with the conventional system alone, the percentage reduction in magnitude of SC currents, and the wave distortion by using fault resistance and Peterson coil.

\section{Types of Grounding Systems}

Selection of a grounding system depends on the application and situation of the systems. Classification of grounding systems is given in Figure 1 [15].

Basically, there are two ways to ground a system, and neutral grounding is the most commonly used. It could be used to ground the whole system or to ground equipment such as generators and transformers [33].

These techniques are applied at a generating station or at a substation. A solid grounding system is directly grounded with no intentional impedance. Most of the low-voltage generators are chosen to be solidly grounded for fast clearing time of high fault currents which can have significant risk to the generator for high-level SLG magnitudes. In a 


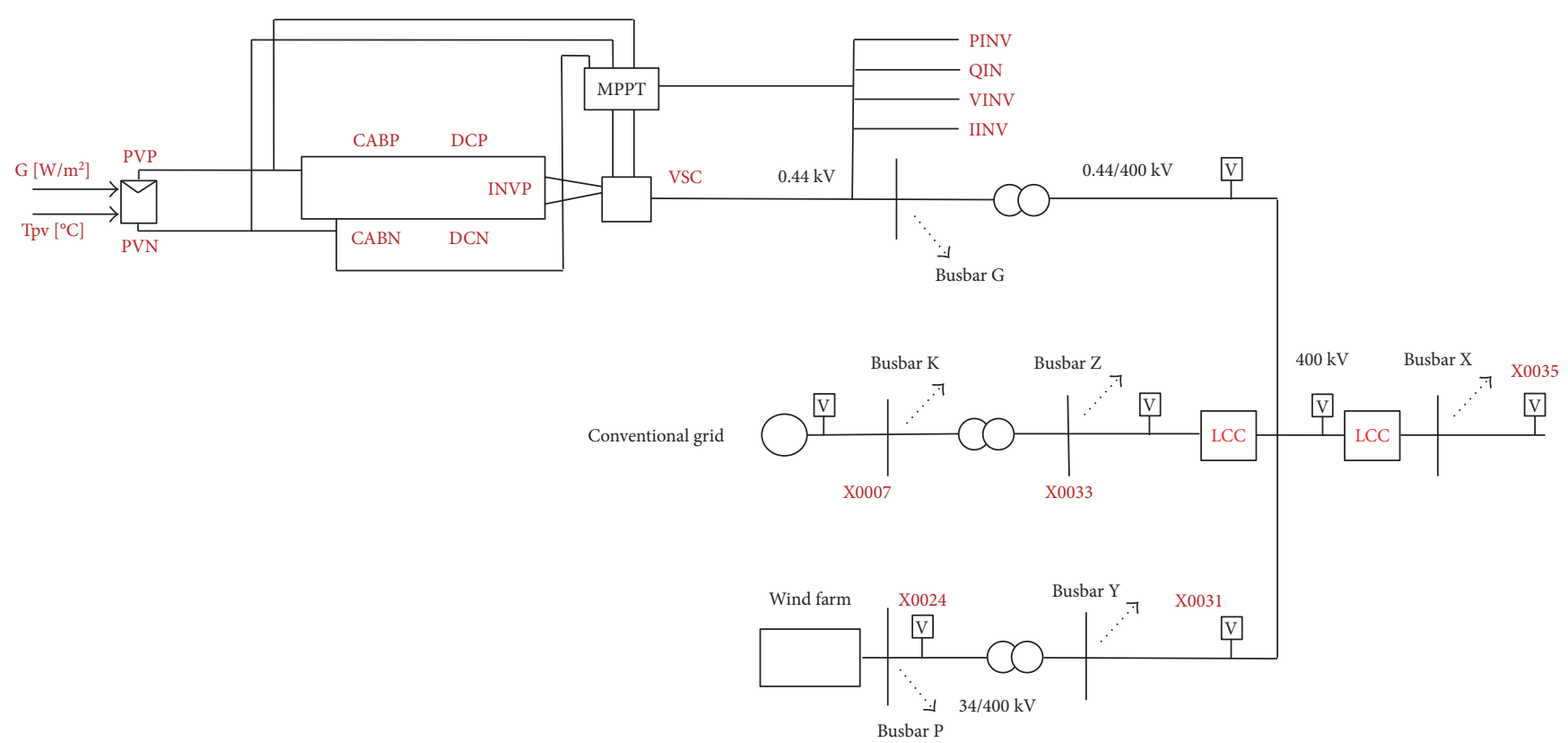

FIGURE 2: No-load wind/PV GCS.

resistance grounding system, a resistor is connected from the source wye point to the ground with a grounding conductor for minimizing fault currents.

Recently, low-resistance grounding is preferred for generators as compared to solid grounding, to increase good reliability against overvoltage and harmonics [15]. A hybrid resistance grounding (HRG) system is most suited in systems with medium-voltage [16] generators. Fault current is reduced by using Petersen coil due to the compensating impact. The system charging and discharging during fault depend on the fault location and the line capacitances to the ground. Fault current compensates through Petersen coil and reduces the capacitive current.

When the SLG fault takes place, the faulted phase discharges current to the ground during fault, which returns through the 2 unfaulted phases [13]. This can elevate the lineto-ground voltages of the 2 unfaulted phases which in turn charge the line-to-ground fault capacitance (insulation).

Resonant coil (Peterson coil) condition is obtained when

$$
3 \omega C_{0}-\frac{1}{\omega L}=0
$$

In the case of complete compensation,

$$
3 L C \omega^{2}=1 \text {. }
$$

\section{Wind/PV Grid-Connected System}

The wind/PV generators are connected with the conventional power system. The $100 \mathrm{MW}$ wind/PV generators are connected with the conventional system at the midpoint of $200 \mathrm{~km}$ OHL at a voltage of $400 \mathrm{kV}$ as shown in Figure 2. In this studied system, a synchronous machine (SM59_NC)type synchronous generator (SG), an induction generator (IG), and 3-phase hybrid transformers (XFMR) are used; one hybrid transformer XFMR is used with SG, that is, $22 / 400 \mathrm{kV}$; the second XFMR is used with a PV generator on the ac side, that is, $0.44 / 400 \mathrm{kV}$ (the interim mediumvoltage systems through which the $\mathrm{PV}$ is fed to get to $400 \mathrm{kV}$ are not mentioned in Figure 2); and the third XFMR [34] is used with IG, that is, $34 / 400 \mathrm{kV}$. All parameters are available in [35].

3.1. SCF Current Controlled by a Fault Resistor. The SCF is considered to take place at busbar $\mathrm{X}$ on the line as shown in Figure 3. The short-circuit fault occurs when the voltage of phase $A$ is at a peak value, that is, at $22.15 \mathrm{msec}$. The waveforms of the fault current at fault location $\mathrm{X}$ are given in Figure 4. Minimization of faulted phase currents at fault location X on the wind/PV GCS due to the SCF for different fault resistances is given in Table 1.

The maximum magnitude of fault currents at fault location $\mathrm{X}$ decreases as the fault resistance increases. Fault current at fault location X decreases from $3.35 \%$ at $1 \Omega$ fault resistance to $88.59 \%$ at $60 \Omega$ fault resistances. It is also observed that SCF current is reduced as fault resistance is increased. Transients in the fault current waveform are higher at a lower value of the fault resistance as compared to a higher value expected. It is also analyzed that the wave attenuation is also higher due to the SCF as compared to the LG fault.

3.2. Comparison between the Wind/PV GCS with Solid Grounding and That with Peterson Coil Grounding under Various Faults. On this wind/PV GCS, different types of faults are considered such as the SLG fault, DLG fault, and SCF. The LG fault occurs on phase A at the end OHL, that is, $400 \mathrm{kV}$. In the case of the DLG fault, the fault occurs at the receiving end of the line on phases $\mathrm{A}$ and $\mathrm{B}$. A comparison between solid grounding and Peterson coil grounding of 


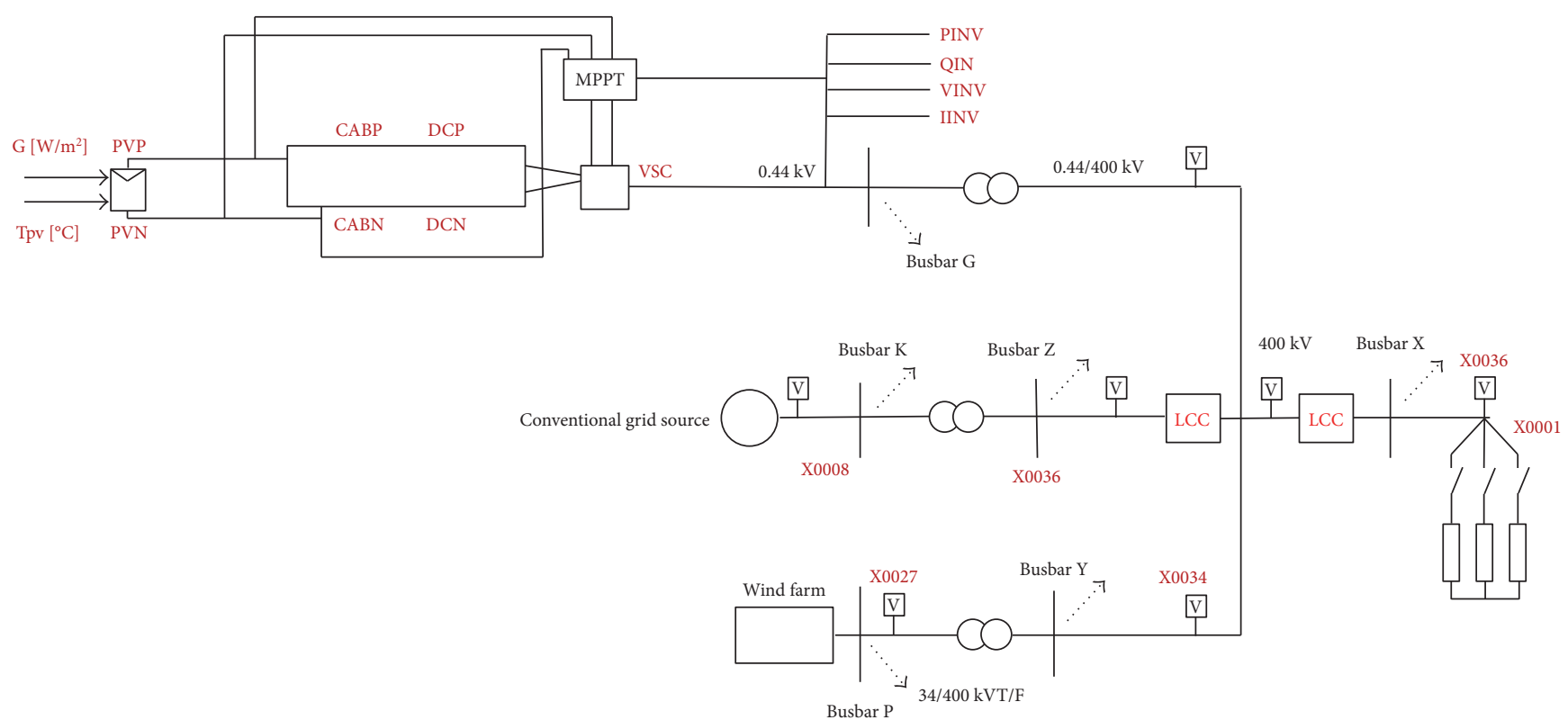

FIGURE 3: Configuration of the studied wind/PV GCS for fault resistance from $1 \Omega$ to $60 \Omega$ with the SCF occurring at busbar X.

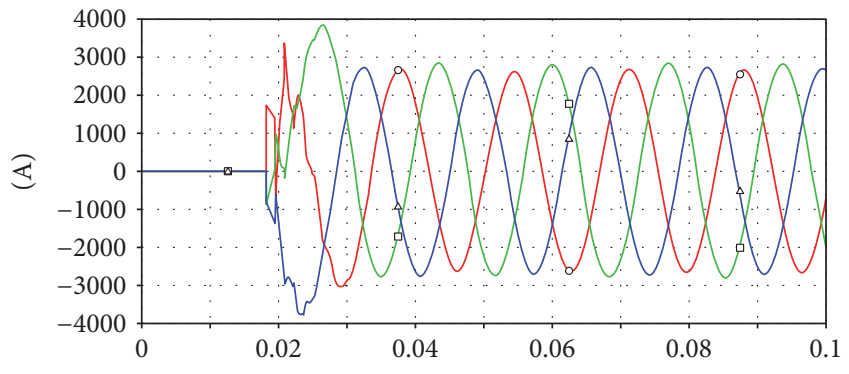

(s)

(file WINDPV100MW_MPPTHYBRID2b.pl4; $\mathrm{x}$-var t) c:X0001A-XX0012 ${ }^{\circ}$ :X0001B-XX0013 ${ }^{\square}$ c:X0001C-XX0014 ${ }^{\Delta}$

FIGURE 4: SCF current at fault location X when the SCF occurs at fault location X with $40 \Omega$ fault resistance on the wind/PV GCS.

TABLE 1: Minimization of faulted phase currents at fault location X the on wind/PV GCS due to the SCF for different fault resistances.

\begin{tabular}{lcccc}
\hline \multicolumn{5}{c}{ Maximum magnitude } \\
Fault resistance \\
$\left(R_{\mathrm{f}}\right)(\Omega)$ & $\begin{array}{c}\text { of fault current (Amp) } \\
\text { SCF }\end{array}$ & $\begin{array}{c}\text { Current Minimization } \\
(\%)\end{array}$ \\
& $\begin{array}{c}\text { Phase } \\
\text { A }\end{array}$ & $\begin{array}{c}\text { Phase } \\
\text { B }\end{array}$ & Phase & C \\
\hline 1 & 5263 & 5976 & 5767 & -3.35 \\
40.58 & 3382 & 3834 & 3791 & -60.85 \\
48 & 3125 & 3583 & 3544 & -72.12 \\
60 & 2801 & 3270 & 3239 & -88.59 \\
\hline
\end{tabular}

$58.6 \mathrm{H}$ inductance on $400 \mathrm{kV}$ windings on the wind/PV GCS under various fault conditions is given in Table 2.

Table 2 shows the SCF currents with solid grounding and with Peterson coil grounding of $58.6 \mathrm{H}$ inductance. SCF current and wave distortion are reduced by using
Peterson coil. Wave distortion is very high when the system is solid grounding as shown in Figures 5 and 6.

Table 2 shows the SLG currents with solid grounding and with Peterson coil grounding of $58.6 \mathrm{H}$ inductance. SLG current is increased to $19.88 \%$, and wave distortion is reduced by using Peterson coil. Wave distortion is very high when the system is solid grounding as shown in Figures 7 and 8. It is also determined that Peterson coil is unsuitable under the SLG fault for $400 \mathrm{kV}$ OHL from a transient overvoltage and distortion perspective. When the system capacitance is matched by the inductance of the coil, the system is fully compensated. If the reactor inductance does not match the system capacitance, the system is off tuned. It can be over- or undercompensated, depending on the relationship between inductance and capacitance. Peterson coil is not suitable for high-voltage and long transmission lines.

Table 2 shows the DLG currents with solid grounding and with Peterson coil grounding of $58.6 \mathrm{H}$ inductance. DLG current and wave distortion are reduced by using Peterson coil. Wave distortion is very high when the system is solid grounding as shown in Figures 9 and 10. It has been analyzed that Peterson coil is most suitable for the SCF and DLG fault. Harmonic distortion occurs due to wind/PV power electronics in Figures 5-10.

3.3. SCF Current Minimization by Using Peterson Coil. The SCF is considered to take place at busbar $\mathrm{X}$ with $R_{\mathrm{f}}=0$ as shown in Figure 11. The short-circuit fault occurs when the voltage of phase $A$ is at a peak value, that is, at $22.15 \mathrm{msec}$. Fault current is reduced by using resonant grounding due to the compensating impact of the Petersen coil. The charging and discharging in the SCF depend on the line-toground capacitances and on the fault location. Fault current through Petersen coil compensates and minimizes the 
TABLE 2: A comparison between solid grounding and Peterson coil grounding of $58.6 \mathrm{H}$ inductance on the wind/PV GCS under various fault conditions.

\begin{tabular}{lccccccc}
\hline & \multicolumn{7}{c}{ Maximum magnitude of fault current at fault location X (Amp) } \\
Fault type & Wind/PV GCS with solid grounding & Wind/PV IGs with Peterson coil & grounding & Current minimization (\%) \\
& Phase A & Phase B & Phase C & Phase A & Phase B & Phase C & -45.28 \\
\hline SCF & 5011 & 6176 & 5832 & 2698 & 4251 & 3985 & 19.88 \\
SLG fault & 2096 & - & - & 2616 & - & - & -43.26 \\
DLG fault & 5119 & 4782 & - & 3208 & 3338 & - & -4 \\
\hline
\end{tabular}

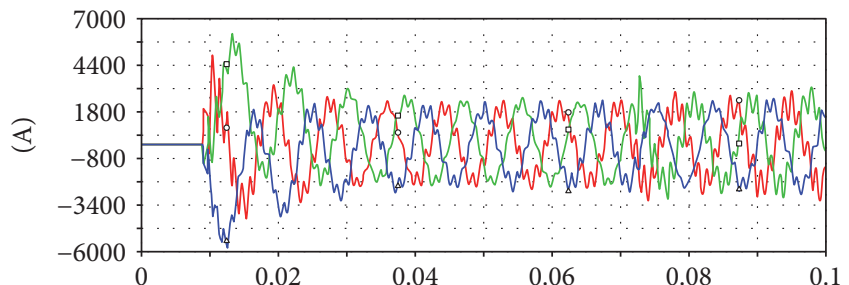

(s)

(file WINDPV100MW_MPPTHYBRID2a.p14; $\mathrm{x}$-var t) c:X0036A-X0001A c:X0036B-X0001B ${ }^{\square}$ c:X0036C-X0001C ${ }^{\Delta}$

FIgURE 5: SCF currents without Peterson coil at fault location X when the SCF occurs on the wind/PV GCS.

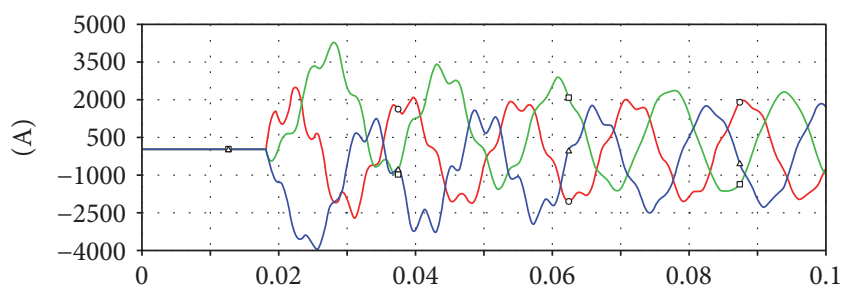

(s)

(file WINDPV100MW_MPPTHYBRID2e.pl4; $\mathrm{x}$-var t ) c:XX0012- ${ }^{\circ}$ :XX0013- ${ }^{-}$c:XX0027- ${ }^{\Delta}$

FIgURe 6: SCF currents with Peterson coil of $58.6 \mathrm{H}$ at fault location $\mathrm{X}$ when the SCF occurs on the wind/PV GCS.

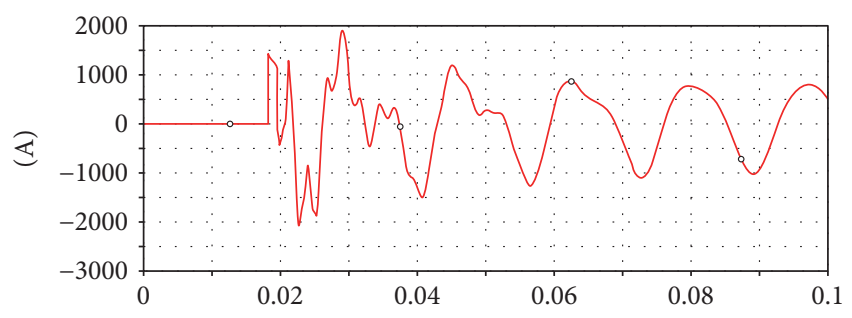

(s)

(file WINDPV100MW_MPPTHYBRID2d.p14; $\mathrm{x}$-var t) c:X0001A

Figure 7: Faulted phase current without Peterson coil at fault location X with the SLG fault on the wind/PV GCS.

capacitive current through the fault location. Table 3 gives the results in the case of Petersen coil with earth fault. Fault current has a small value because of the high percentage of

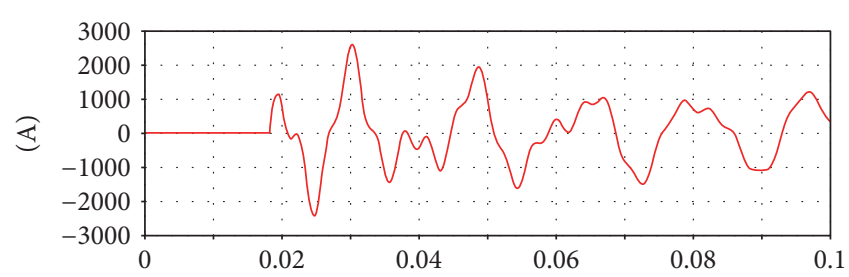

(s)

(file WINDPV100MW_MPPTHYBRID2g.pl4; $\mathrm{x}$-var t) c:X0012-

FIgURE 8: Faulted phase current with Peterson coil of $58.6 \mathrm{H}$ at fault location X with the SLG fault on the wind/PV GCS.

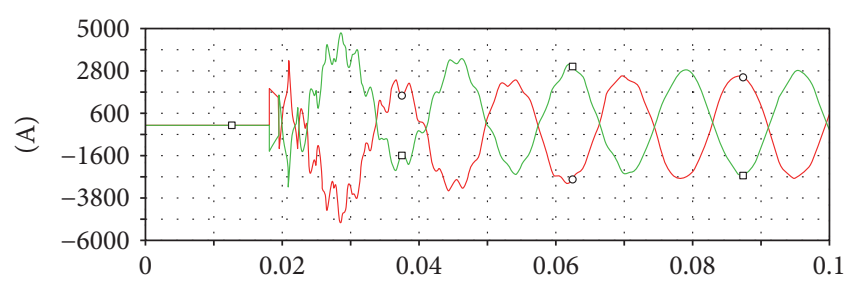

(s)

(file WINDPV100MW_MPPTHYBRID2a.p14; $\mathrm{x}$-var t) c:X0001A c:X0001B

FIgURE 9: Current of faulted phases A and B without Peterson coil at fault location X when the DLG fault occurs on the wind/PV GCS.

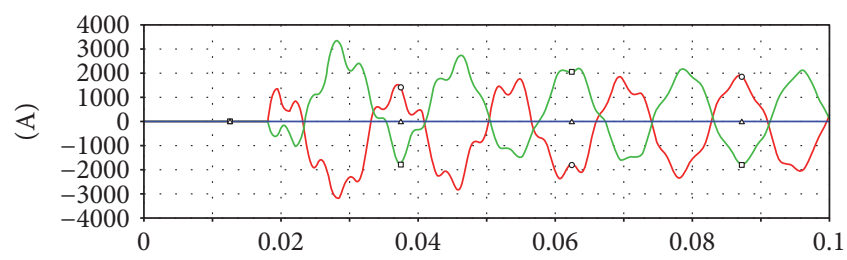

(s)

(file WINDPV100MW_MPPTHYBRID2g.pl4; $\mathrm{x}$-var t) c:X0037A-X0001A c:X0037B-X0001B ${ }^{\square}$ c:X0037C-X0001C ${ }^{4}$

Figure 10: Current of faulted phases A and B with Peterson coil of $58.6 \mathrm{H}$ at fault location $\mathrm{X}$ when the DLG fault occurs on the wind/PV GCS.

compensation. This small value of fault current enables the system to continue feeding the consumers under fault for a long time. To compensate for the capacitive earth fault current of the feeder (with $200 \mathrm{~km}$ ), the zero sequence capacitance is approximately $0.0079 \mu \mathrm{F} / \mathrm{km}$. 


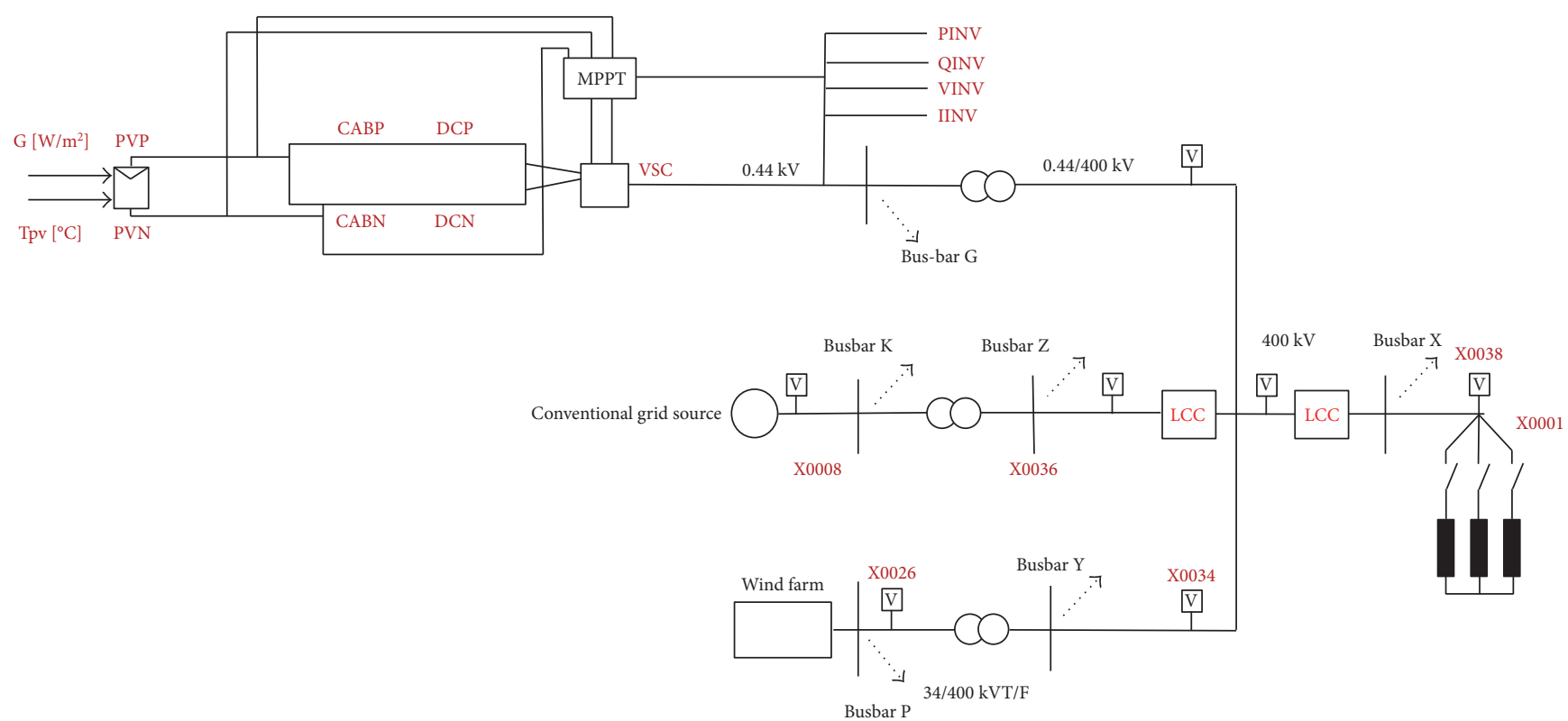

FIgure 11: Configuration of the studied wind/PV GCS when Peterson coil varies from $1 \mathrm{H}$ to $65 \mathrm{H}$ with the SCF occurring at busbar X.

TABLE 3: Minimization of fault currents at fault location $\mathrm{X}$ on the wind/PV GCS due to the SCF for different values of Peterson coil.

\begin{tabular}{lcccc}
\hline \multirow{2}{*}{$\begin{array}{l}\text { Fault inductance } \\
\left(L_{\mathrm{f}}\right)(\mathrm{H})\end{array}$} & \multicolumn{4}{c}{$\begin{array}{l}\text { Maximum magnitude of fault } \\
\text { current }\end{array}$} \\
\hline 1 & Phase A & Phase B & Phase C & \\
\hline 10 & 5318 & 6154 & 5810 & 0.36 \\
30 & 4349 & 5733 & 5420 & 7.73 \\
58.6 & 3457 & 5111 & 4883 & 20.84 \\
65 & 2698 & 4251 & 3985 & 45.28 \\
\hline
\end{tabular}

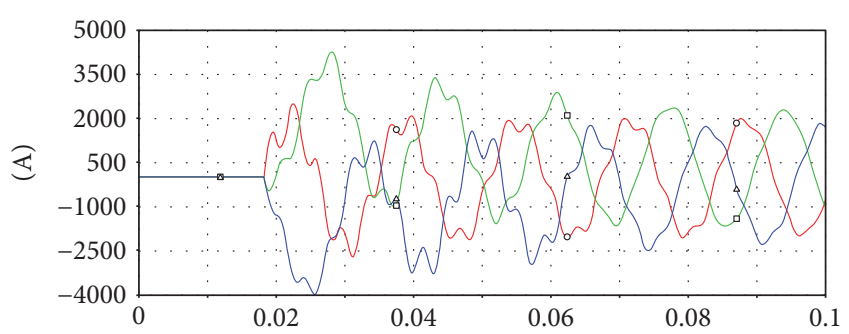

(s)

(file WINDPV100MW_MPPTHYBRID2f.pl4; $\mathrm{x}$-var t) c:XX0012- ${ }^{\circ}$ c:XX0013- ${ }^{\square}$ c:XX0027- ${ }^{\Delta}$

FIGURE 12: SC current at fault location X when the SCF occurs at fault location $\mathrm{X}$ with $58.6 \mathrm{H}$ fault inductance on the wind/PV GCS.

Minimization of fault currents at fault location $\mathrm{X}$ on the wind/PV GCS due to the SCF for different values of Peterson coil is given in Table 3 . The waveforms of the fault current at fault location $\mathrm{X}$ are given in Figure 12. The maximum magnitude of fault currents at fault location $\mathrm{X}$ decreases as the fault inductance increases. Fault current at fault

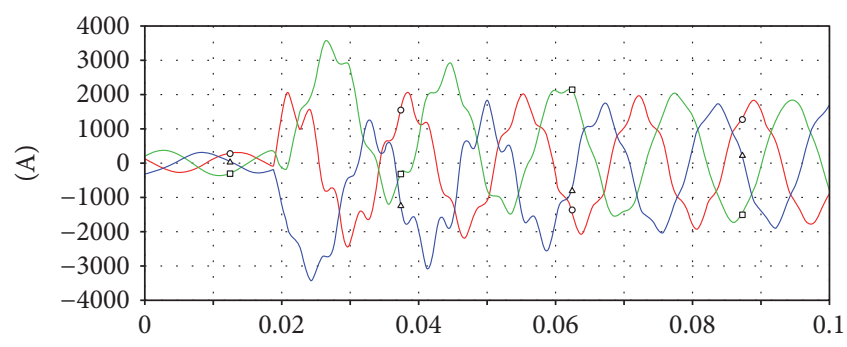

(s)

(file WINDPV100MW_MPPTHYBRID2f.pl4; x-var t) c:X0036A-X0033A c:X0036B-X0033B ${ }^{\square}$ c:X0036C-X0033C ${ }^{\Delta}$

Figure 13: SC current at fault location $\mathrm{X}$ when the SCF occurs at fault location $\mathrm{X}$ with $58.6 \mathrm{H}$ fault inductance on the wind/PV GCS (it is a not shunt-connected inductor).

location $\mathrm{X}$ decreases from $0.36 \%$ at $1 \mathrm{H}$ fault inductance to $51.78 \%$ at $65 \mathrm{H}$ fault inductance. Transients in the fault current waveform are higher at a lower value of the fault inductance as compared to a higher value. It is also analyzed that the wave attenuation is also higher due to the SCF.

The waveforms of the fault current at busbar $\mathrm{Z}$ are given in Figure 13. Minimization of fault currents at busbar $\mathrm{Z}$ on the wind/PV GCS due to the SCF for different values of Peterson coil is given in Table 4. The maximum magnitude of fault currents at busbar $\mathrm{Z}$ decreases as the fault inductance increases. Fault current at fault location $\mathrm{X}$ decreases from $3.69 \%$ at $1 \mathrm{H}$ fault inductance to $47.79 \%$ at $65 \mathrm{H}$ fault inductance. Transients in the fault current waveform are higher at a lower value of the fault inductance as compared to a higher value. It is also analyzed that the wave attenuation is also higher due to the SCF.

Maximum magnitude of the voltage of faulted and unfaulted phases at fault location $\mathrm{X}$ when SCFs occur with 
TABLE 4: Minimization of fault currents at busbar $\mathrm{Z}$ on the wind/PV GCS due to the SCF for different values of Peterson coil.

\begin{tabular}{lcccc}
\hline \multicolumn{4}{c}{$\begin{array}{l}\text { Maximum magnitude } \\
\text { of fault current with }\end{array}$} & $\begin{array}{c}\text { Current } \\
\left(L_{\mathrm{f}}\right)(\mathrm{H})\end{array}$ \\
& \multicolumn{2}{c}{ the SCF (Amp) } & minimization $(\%)$ \\
& Phase & Phase & Phase & \\
\hline 1 & A & B & C & \\
10 & 3356 & 4980 & 4801 & -3.69 \\
30 & 3061 & 4700 & 4495 & -9.87 \\
58.6 & 2796 & 4116 & 3850 & -25.46 \\
65 & 2466 & 3565 & 3439 & -44.85 \\
\hline
\end{tabular}

TABle 5: Maximum magnitude of the voltage of faulted and unfaulted phases at fault location X when the SCF occurs with fault inductance of Peterson coil varying from $1 \mathrm{H}$ to $65 \mathrm{H}$ on wind/PV IGs.

\begin{tabular}{|c|c|c|c|}
\hline \multirow[t]{2}{*}{ Fault inductance $(\mathrm{H})$} & \multicolumn{3}{|c|}{$\begin{array}{c}\text { Maximum magnitude of the voltage of } \\
\text { unfaulted phases at fault location X } \\
\text { with Peterson coil (MV) }\end{array}$} \\
\hline & Phase A & Phase B & Phase C \\
\hline 1 & 0.00611 & 0.00237 & 0.002568 \\
\hline 58.6 & 0.2808 & 0.2369 & 0.2843 \\
\hline 65 & 0.2961 & 0.2428 & 0.3059 \\
\hline
\end{tabular}

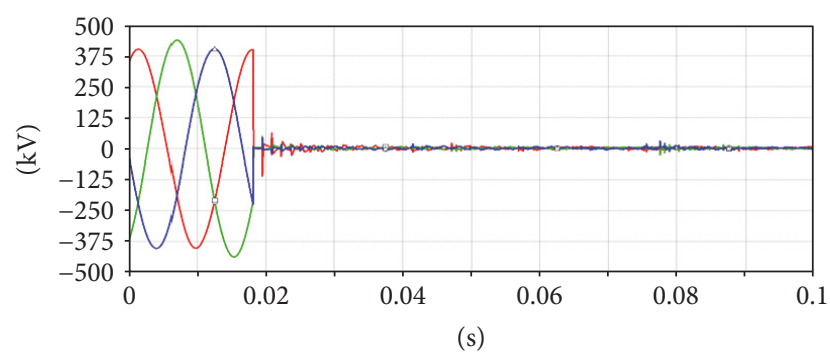

(file WINDPV100MW_MPPTHYBRID2e.pl4; $\mathrm{x}$-var t)

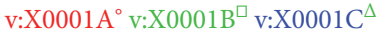

FIgURE 14: SC voltage at fault location $\mathrm{X}$ when the SCF occurs at fault location $\mathrm{X}$ with $1 \mathrm{H}$ fault inductance on wind/PV IGs.

fault inductance of Peterson coil that varies from $1 \mathrm{H}$ to $65 \mathrm{H}$ on the wind/PV GCS is given in Table 5 .

The voltage of faulted phases increased from $6.11 \mathrm{kV}$ at $1 \mathrm{H}$ to $296.1 \mathrm{kV}$ at $65 \mathrm{H}$ inductances because it also acts as the voltage source. Wave attenuation is also increased as inductance increased as shown in Figures 14 and 15. It is observed that fault currents due to the SLG fault are less as compared to those due to the SCF on wind/PV IGs. It means that the SCF has severe effects as compared to the SLG fault on the wind/PV hybrid system, when the effect of fault inductance is studied. It is also analyzed that the wave attenuation is also higher due to the SCF as compared to the SLG fault.

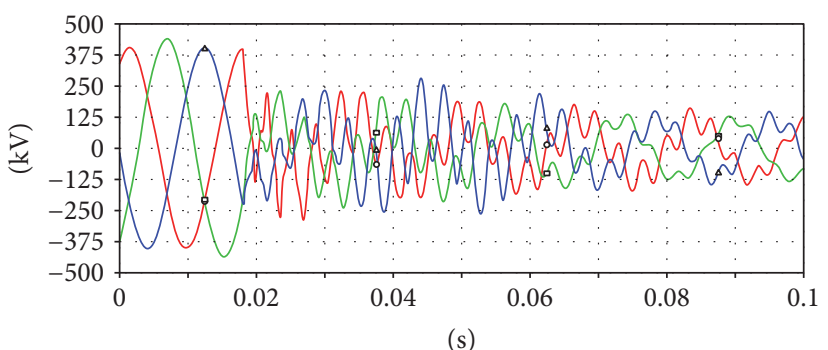

(file WINDPV100MW_MPPTHYBRID2a.pl4; $\mathrm{x}$-var t) c:X0036A-X0001A c:X0036B-X0001B c:X0036C-X0001C ${ }^{\square}$

Figure 15: SC voltage at fault location $\mathrm{X}$ when the SCF occurs at fault location $\mathrm{X}$ with $58.6 \mathrm{H}$ fault inductance on wind/PV IGs.

\section{Conclusion}

This investigation is significant for protection, designing, planning, and operation of the wind/PV GCS. The waveforms are analyzed for SCF current, which are used for designing the protection strategy. This research presents the transient overcurrent and overvoltage responses due to various faults, and the protection schemes such as solid, resistance, and Peterson coil grounding are examined for minimizing fault currents. These features have a significant contribution to the magnitudes and shapes of the current and voltage waveforms along OHL.

This research included three types of faults including the SCF, SLG fault, and DLG fault. A detailed comparison between three cases has been examined, and this research was on a conventional system alone with the wind/PV GCS. The wind/PV generator had the most severe impact on the SCF. Fault calculation models are completed in ATP/EMTP software for HV wind/PV GCSs. The models investigate SCFs under various grounding methods and fault factors. SCF, SLG fault, and DLG fault currents are controlled by varying the value of fault resistance. The capacitive charging currents in OHL contribute to the fault current. Voltages of unfaulted phases are higher than the rated level of voltage.

When the SCF is grounded through fault resistance, then, SCF current is decreased from $3.35 \%$ at $1 \Omega$ to $88.59 \%$ at $60 \Omega$ fault resistance. Wave attenuation is also decreased as fault resistance increased from a low value of fault resistance to a high value.

Fault currents with solid grounding and with Peterson coil grounding of $58.6 \mathrm{H}$ inductance under various faults are also observed. SCF current is reduced to $45.28 \%$, and wave distortion in fault current is also reduced by using Peterson coil grounding. SLG current is increased to $19.88 \%$, and wave distortion is reduced by using Peterson coil grounding. DLG current is reduced to $43.26 \%$ and wave distortion is also reduced by using Peterson coil grounding. But wave distortions are very high for SCF, SLG, and DLG states, when the system is solid grounding. It has been analyzed that Peterson coil is most suitable for the SCF and DLG fault. In North America, the Peterson coil would only be considered for SLG purposes. Due to high voltage in OHL, it would 
require hundreds of higher voltage insulators over $\mathrm{km}$; thus, it is never selected.

Minimization of fault currents at fault location $\mathrm{X}$ and busbar Z on the wind/PV GCS due to the SCF for different values of Peterson coil at fault location $\mathrm{X}$ is also examined. The maximum magnitude of fault currents at fault location $\mathrm{X}$ decreases as the fault inductance increases. Fault current at fault location $\mathrm{X}$ decreases from $0.36 \%$ at $1 \mathrm{H}$ fault inductance to $51.78 \%$ at $65 \mathrm{H}$ fault inductance. Transients in the fault current waveform are higher at a lower value of the fault inductance as compared to a higher value. The maximum magnitude of fault currents at busbar $\mathrm{Z}$ decreases as the fault inductance increases. Fault current at busbar $\mathrm{Z}$ decreases from $3.69 \%$ at $1 \mathrm{H}$ fault inductance to $47.79 \%$ at $65 \mathrm{H}$ fault inductance. Transients in the fault current waveform are higher at a lower value of the fault inductance as compared to a higher value. It is also analyzed that the wave attenuation is also higher due to the SCF.

\section{Conflicts of Interest}

The authors declare that they have no conflicts of interest.

\section{References}

[1] J. M. Carrasco, L. G. Franquelo, J. T. Bialasiewicz et al., "Power-electronic systems for the grid integration of renewable energy sources: a survey," IEEE Transactions on Industrial Electronics, vol. 53, no. 4, pp. 1002-1016, 2006.

[2] R. Chedid and S. Rahman, "Unit sizing and control of hybrid wind-solar power systems," IEEE Transactions on Energy Conversion, vol. 12, no. 1, pp. 79-85, 1997.

[3] T. N. Boutsika and S. A. Papathanassiou, "Short-circuit calculations in networks with distributed generation," Electric Power Systems Research, vol. 78, no. 7, pp. 1181-1191, 2008.

[4] H. R. Baghaee, M. Mirsalim, M. J. Sanjari, and G. B. Gharehpetian, "Effect of type and interconnection of DG units in the fault current level of distribution networks," in 2008 13th International Power Electronics and Motion Control Conference, pp. 313-319, Poznan, Poland, September 2008.

[5] S. Boljevic and M. F. Conlon, "The contribution to distribution network short-circuit current level from the connection of distributed generation," in 2008 43rd International Universities Power Engineering Conference, pp. 1-6, Padova, Italy, September 2008.

[6] S. Barghi, M. A. Golkar, and A. Hajizadeh, "Impacts of distribution network characteristics on penetration level of wind distributed generation and voltage stability," in 2011 10th International Conference on Environment and Electrical Engineering, pp. 1-4, Rome, Italy, May 2011.

[7] J. A. Martinez-Velasco, Transient Analysis of Power Systems: Solution Techniques, Tools and Applications, John Wiley \& Sons Ltd., United Kingdom, 2014.

[8] L. van der Sluis, Transients in Power Systems, John Wiley \& Sons Ltd., United Kingdom, 2001.

[9] M. Parkkonen, "The use of EMTPIATP program in induction generator connection studies to the electrical network," in Minutes of the 23rd EMTP User Group Meeting, Lisbon, Portugal, June 1993.
[10] IEEE, STANDARD 142-2007-IEEE Recommended Practice for Grounding of Industrial and Commercial Power Systems (Green Book), IEEE, USA, 2007.

[11] IEEE, STANDARD 1100-2005-IEEE Recommended Practice for Powering and Grounding Electronic Equipment (Emerald Book), IEEE, USA, 2006.

[12] D. D. Shipp and F. J. Angelini, "Characteristics of different power systems neutral grounding techniques: fact and fiction," in IEEE Textile, Fiber and Film Industry Technical Conference 1991, Greenville, SC, USA, May 1991.

[13] A. R. Al-Zyoud, A. Alwadie, A. Elmitwally, and A. Basheer, "Effect of neutral grounding methods on the earth fault characteristics," in PIERS Proceedings, pp. 1144-1151, Prague, Czech Republic, July 2015.

[14] M. Pühringer, Resonant Grounding as Approach to System Neutral Grounding, Haefely Trench, Austria, 1998.

[15] D. Jacob and K. Nithiyanthan, "Effective methods for power systems grounding," WSEAS Transactions on Business and Economics, vol. 5, no. 5, pp. 151-160, 2008.

[16] P. Pillai, A. Pierce, B. Bailey et al., "Grounding and ground fault protection of multiple generator installations on medium-voltage industrial and commercial systems, part 2: grounding methods," in Conference Record of the 2002 IEEE Industry Applications Conference. 37th IAS Annual Meeting (Cat. No.02CH37344), vol. 3, pp. 1888-1895, Pittsburgh, PA, USA, October 2002.

[17] A. Bapat, R. Hanna, and S. Panetta, "Advanced concepts in high resistance grounding," in Petroleum and Chemical Industry Technical Conference (PCIC), 2012 Record of Conference Papers Industry Applications Society 59th Annual IEEE, pp. 1-9, Chicago, IL, USA, September 2012.

[18] M. T. Al-Hajri, "Neutral ground resistor monitoring schemes," in 2004 Conference Record of the IEEE International Symposium on Electrical Insulation, Indianapolis IN, USA, September 2004.

[19] J. Roberts, H. J. Altuve, and D. Hou, Review of Ground Fault Protection Methods for Grounded, Ungrounded, and Compensated Distribution Systems, Schweitzer Engineering Laboratories, Inc., Pullman, WA USA, 2001.

[20] T. Welfonder and V. Leitloff, "Location strategies and evaluation of detection algorithms for earth faults in compensated MV distribution systems," IEEE Transactions on Power Delivery, vol. 15, no. 4, pp. 1121-1128, 2000.

[21] D. Griffel, Y. Harmand, V. Leitloff, and J. Bergeal, "A new deal for safety and quality on MV networks," IEEE Transactions on Power Delivery, vol. 12, no. 4, pp. 1428-1433, 1997.

[22] M. Abdel-Fattah, "A transient fault detection technique with varying fault detection window of earth modes in unearthed MV systems," in 2008 Power Quality and Supply Reliability Conference, Parnu, Estonia, August 2008.

[23] L. F. Hunt and J. H. Vivian, "Sensitive ground protection for radial distribution feeders," Transactions of the American Institute of Electrical Engineers, vol. 59, pp. 84-90, 1940.

[24] I. Zamora, A. J. Mazon, F. Antepara, M. Puhringer, and J. R. Saenz, "Experiences of neutral resonant system implementation in Gorliz substation," in CI RED 17th International Conference on Electricity Distribution, Barcelona, May 2003.

[25] R. Van de Sandt, J. Lowen, J. Paetzold, and I. Erlich, "Neutral earthing in off-shore wind farm grids," in 2009 IEEE Bucharest PowerTech, Bucharest, Romania, June-July 2009. 
[26] S. H. Dumitru Toader, C. Blaj, and I. Cata, Numerical Simulation of Single Phase Faults in Medium Voltage Electrical Networks, University of Timisoara, Romania, 2010.

[27] A. K. Gernot Druml and O. Seifert, "A new directional transient relay for high ohmic earth faults," in CI RED 17th International Conference on Electricity Distribution, Barcelona, Spain, May 2003.

[28] T. Henriksen and A. Petteteig, "Detection of earth fault in a medium voltage distribution network," in International Conference on Power System Transients, Lyon, France, June 2007.

[29] A. Cerretti, F. M. Gatta, A. Geri, S. Lauria, M. Maccioni, and G. Valtorta, "Temporary overvoltages due to ground faults in MV networks," in 2009 IEEE Bucharest PowerTech, Bucharest, Romania, June-July 2009.

[30] A. B. S. Mariappan, M. Rayees, and M. AlDahmi, "Earthing system analysis to improve protection system performance in distribution networks," in 12th IET International Conference on Developments in Power System Protection (DPSP 2014), pp. 1-6, Copenhagen, Denmark, March-April 2014.

[31] P. Toman, J. Dvorak, J. Orsagova, and S. Misak, "Experimental analysis of electrical values during earth faults," in Proceedings of the 2010 Electric Power Quality and Supply Reliability Conference, pp. 185-190, Kuressaare, Estonia, June 2010.

[32] M. Lehtoneen, "Characteristics of earth faults in electrical distribution networks with high impedance earthing," Electric Power Systems Research, vol. 44, no. 3, pp. 155-161, 1998.

[33] IEEE, C37.101-1993-IEEE Guide for Generator Ground Protection, IEEE, USA, 1994.

[34] B. A. Mork, F. Gonzalez, D. Ishchenko, D. L. Stuehm, and J. Mitra, "Hybrid transformer model for transient simulationpart I: development and parameters," IEEE Transactions on Power Delivery, vol. 22, pp. 248-255, 2007.

[35] F. Umer, N. Cetinkaya, and G. S. Shehu, "Transient analysis of short circuit faults in PV hybrid systems," in EEETEM IEEE Conference, pp. 28-35, Beirut, Lebanon, April 2017. 

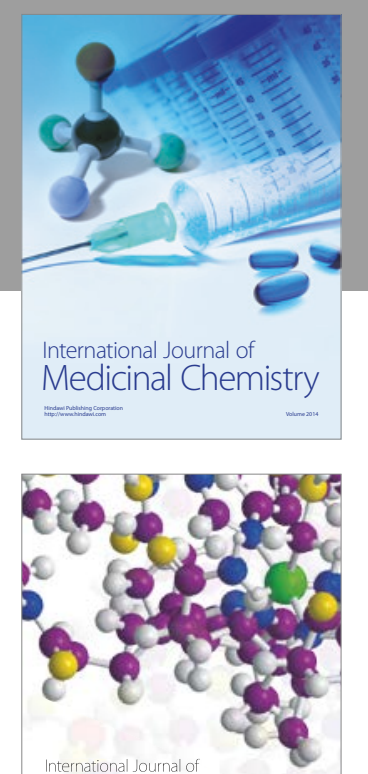

Carbohydrate Chemistry

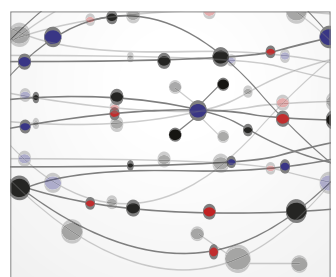

The Scientific World Journal
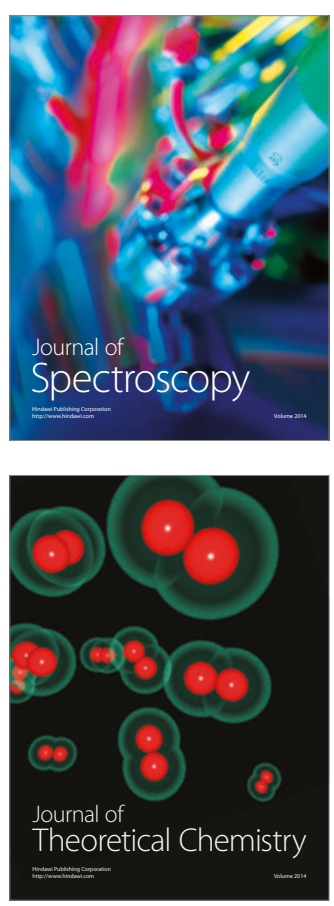
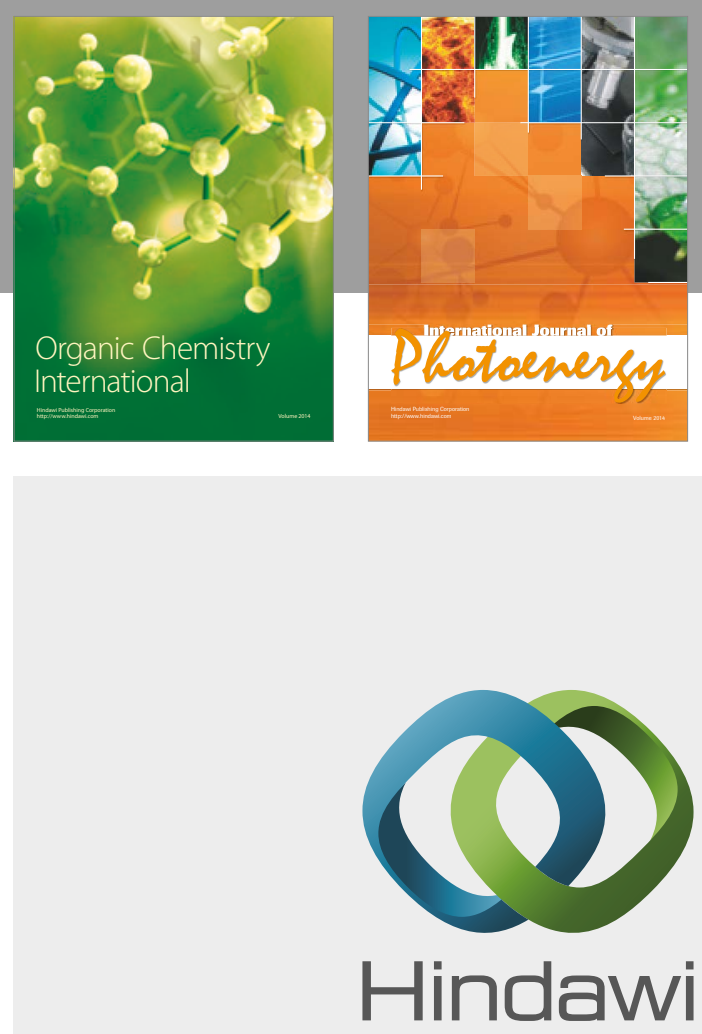

Submit your manuscripts at

https://www.hindawi.com

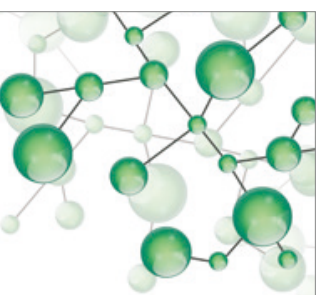

International Journal of

Inorganic Chemistry

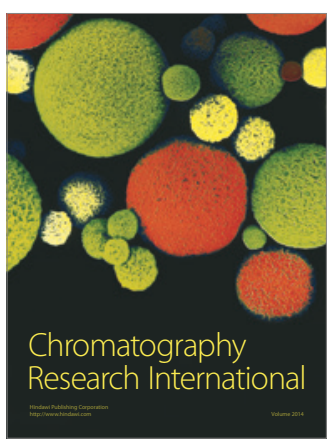

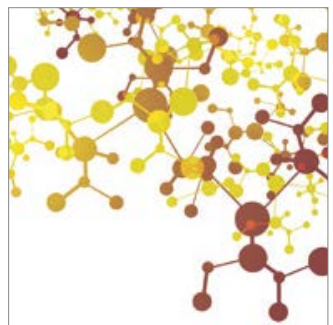

Applied Chemistry
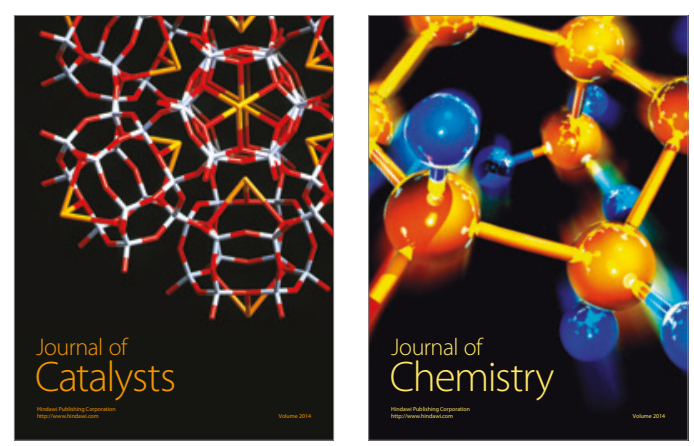
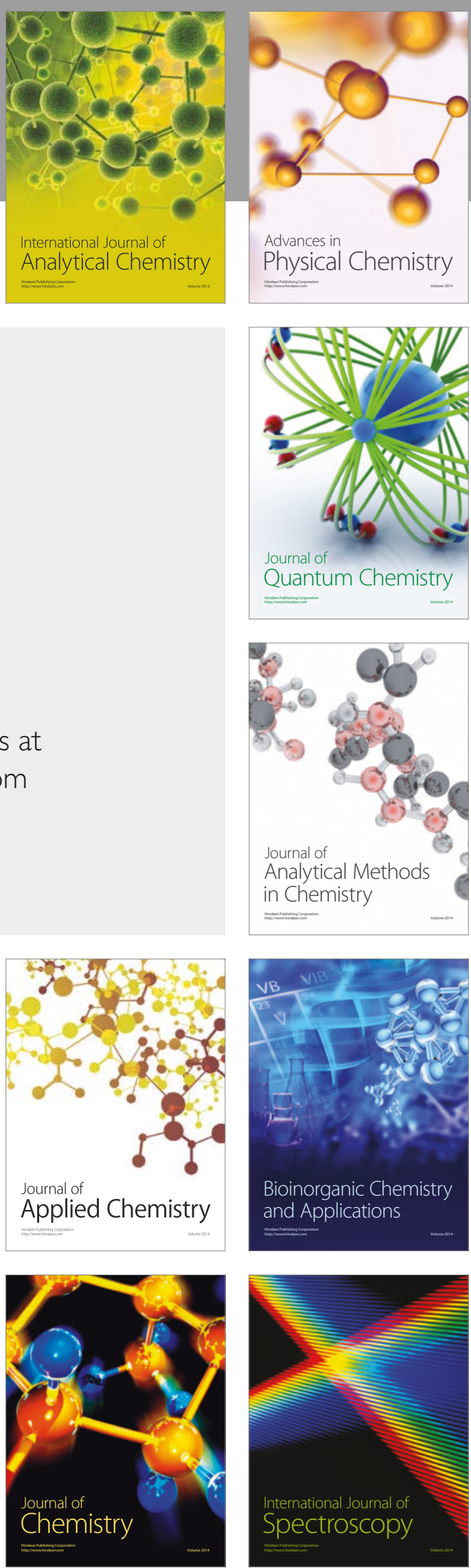